\title{
Topological Defects in Electric Double Layers of Ionic Liquids at Carbon Interfaces
}

Jennifer M. Black, M. Baris Okatan, Guang Feng, ${ }^{*}$ Peter T. Cummings, Sergei V. Kalinin, and Nina Balke*

Dr. J. M. Black

Dr. M. B. Okatan

Dr. S. V. Kalinin

Dr. N. Balke (corresponding author experimental - balken@ornl.gov)

Center for Nanophase Materials Sciences, Oak Ridge National Laboratory, Oak Ridge, TN, 37831, USA

Prof. G. Feng (corresponding author theory - gfeng@ @ust.edu.ch )

State Key Laboratory of Coal Combustion, School of Energy and Power Engineering, Huazhong University of Science and Technology (HUST), Wuhan 430074, China

Prof. P. T. Cummings

Department of Chemical \& Biomolecular Engineering and Multiscale Modeling and Simulation Center, Vanderbilt University, Nashville, TN, 37235, USA 


\begin{abstract}
The structure and properties of the electrical double layer in ionic liquids is of interest in a wide range of areas including energy storage, catalysis, lubrication, and many more. Theories describing the electrical double layer for ionic liquids have been proposed, however a full molecular level description of the double layer is lacking. To date, studies have been predominantly focused on ion distributions normal to the surface, however the 3D nature of the electrical double layer in ionic liquids requires a full picture of the double layer structure not only normal to the surface, but also in plane. Here we utilize 3D force mapping to probe the in plane structure of an ionic liquid at a graphite interface and report the direct observation of the structure and properties of topological defects. The observation of ion layering at structural defects such as step-edges, reinforced by molecular dynamics simulations, defines the spatial resolution of the method. Observation of defects allows for the establishment of the universality of ionic liquid behavior vs. separation from the carbon surface and to map internal defect structure. These studies offer a universal pathway for probing the internal structure of topological defects in soft condensed matter on the nanometer level in three dimensions.
\end{abstract}

\title{
Keywords: ionic liquid, carbon, electric double layer, defect, atomic force microscopy
}

\subsection{Introduction}

The electrical double layer formed at the electrode-electrolyte interface is central to many physical and chemical processes. Traditionally the electrical double layer is described using classical models which were developed for dilute electrolytes such as Gouy-Chapman. More 
recently, ionic liquids have emerged as a new class of electrolytes with unique properties such as low vapor pressure, wide potential window, high thermal stability, high ionic conductivity, and low flammability. [1] This has motivated researchers to develop a new picture of the electrical double layer for these highly concentrated ionic systems as the classical models are not suitable to describe this class of materials. [2] Theories describing the electrical double layer for ionic liquid electrolytes have been proposed including over-screening and crowding, however a full molecular level description of the double-layer is still required.[3] Ionic liquids form alternating layers of anions and cations extending several $\mathrm{nm}$ from the interface, akin to the smectic phase of liquid crystals (LC), as evidenced in numerous experimental and computational studies.[4-22] It is crucial to have a full molecular scale picture of the electrical double layer for advances to be made in the numerous areas of application for ionic liquids including energy storage, catalysis, lubrication and many others. [23] Previous studies have mainly focused on ion distributions normal to the electrode surface, however several recent publications highlight the importance of understanding the 3D structure of the double layer in ionic liquids.[24, 25] Previous experimental and simulation results have shown in-plane ordering of the ions at the surface which has been linked to electrochemical response.[24, 26, 27] Therefore it is important to have techniques capable of determining the interfacial structure of ionic liquids at the molecular level in a 3D nature. Scanning probe techniques are well suited for this type of study due to their high lateral resolution, and scanning tunneling microscopy (STM) and AFM have been used to image the $2 \mathrm{~d}$ molecular level structure of the adsorbed layer of ionic liquids on various substrates[9, 28, 29] and has also been extended to image near surface layers providing information in 3dimensions.[30] Similarly 3D mapping of liquid structure at the solid-liquid interface has been done for aqueous electrolytes on graphite,[31] mica,[32] and $\mathrm{Al}_{2} \mathrm{O}_{3}[33]$ surfaces. Additionally, 
multiple force distance studies have been published which provide information on the ionic liquid structure normal to the surface, however these studies have been confined to a single location providing information in only 2 dimensions.[4-12]

In general, the properties of soft condensed matter systems, such as liquid crystals, are coupled to the partial long range ordering and the structure can be strongly affected on macroscopic lengths scales by the presence of interfaces, inclusions, and defects. The properties will be influenced by the structure and dynamics of topological defects, their response to external fields, and interaction with other defects. Examples include the dislocations and disclination lines in liquid crystals that control their optical properties, vortices in superconductors that control electronic transport, and many others. Therefore, it can be expected that defects in the ionic liquid structure at the solid-liquid interface will similarly affect properties, such as charge storage. This emphasizes the need and importance of studying the 3D structure of electric double layers.

Here, we utilize 3D force mapping to probe the in-plane structure of the ordered ionic liquid layers at a highly oriented pyrolytic graphite (HOPG) surface and explore the interactions between ordering and structural defects and topological defects. In this approach, the forcedistance curves are obtained in a dense array, visualizing the $3 \mathrm{D}$ structure of the force field above the material surface. For each point, enough force distance curves were measured to guarantee statistical significance as described in our previous work.[4] It is important to note that in this study measurements are taken over length scales of $500-1000 \mathrm{~nm}$, much larger than the length scales investigated in Ref. [30] $(<5 \mathrm{~nm})$ which investigated the atomic level structure of ionic liquids at the interface, and therefore we are able to image features of the ionic liquid structure occurring over much larger length scales. Using structural defects defined by 
topographic step-edges combined with molecular dynamics (MD) simulations, we establish the lateral resolution of the method. We further observe the presence of dislocation-like topological defects, and map their internal structure and interplay between phase shift and degree of ordering. The universality of ordering as a function of surface separation is observed. These studies provide first visual insight into internal structure of topological defects in soft condensed matter, and open broad opportunities to study a wide range of systems including colloids, liquid crystals, and ionic liquids whose physical properties are determined by structure and dynamics at mesoscopic scales. Moreover it would be interesting to examine how the presence of topological defects within the electrical double layer affects electrochemical properties such as capacitance and charging dynamics.

\subsection{Methods}

\section{Experimental:}

Force-distance curves were collected in contact mode on a Cypher AFM from Asylum Research (Santa Barbara, CA) under ambient conditions in a droplet of the room temperature ionic liquid 1-ethyl-3methyl-imidazolium bis(trifluoromethanesulfonyl)imide $\left(\right.$ Emim $\left.^{+} \mathrm{Tf}_{2} \mathrm{~N}^{-}\right)$as received from the supplier (Boulderionics, Arvada, $\mathrm{CO}$ ) on a freshly cleaved highly oriented pyrolytic graphite (HOPG) surface. Water content was determined by Karl Fisher titration to be $5.7 \mathrm{ppm}$ as received and $6.3 \mathrm{ppm}$ after $24 \mathrm{~h}$ exposure to atmosphere. Curves were collected at a rate of $0.25 \mathrm{~Hz}$, and 50 curves were collected at each location. Experiments were performed using either bare or Au coated silicon nitride tips with a spring constant of ca. $0.6 \mathrm{~N} / \mathrm{m}$. Tips were calibrated using the thermal tune method. In order to compare all curves and account for drift, the curves were aligned along the y-axis to be at zero force far away from the sample. In 
addition, the curves were shifted along the $\mathrm{x}$-axis so that the retract branch of all force curves

overlaid. The tip-sample separation was calculated by subtracting the cantilever deflection from the $\mathrm{z}$ piezo position.

\section{Simulation:}

MD simulation system consists of ionic liquid $\left[\mathrm{Emim}^{+}\right]\left[\mathrm{Tf}_{2} \mathrm{~N}^{-}\right]$enclosed between two channel walls as electrodes, and each electrode was modeled by three layers of graphene sheets with an area of $4.2 \times 4.2 \mathrm{~nm}^{2}$ and a gap of $0.34 \mathrm{~nm}$ between each two layers. The inner graphene (i.e., the one contacting with electrolyte) was cut as half of $4.2 \times 4.2 \mathrm{~nm}^{2}$ with either zigzag or armchair edge. The separation between two electrodes was set to $8.0 \mathrm{~nm}$ (the distance between the innermost non-step layers of opposing channel walls) to reproduce a bulk-state behavior of ILs in the channel center. The force fields for the electrode atoms (carbon) and ions in $\left[\mathrm{Emim}^{+}\right]\left[\mathrm{Tf}_{2} \mathrm{~N}^{-}\right]$ were taken from Ref. [40]. Simulations were performed in the NVT ensemble using a customized MD code based on the Gromacs software. [41] The IL temperature was maintained at a setting value using the Nosé-Hoover thermostat. The number of IL molecules inside the channel was adjusted so that the IL density in the channel center matched that of the bulk IL. The slab-PME method[42] was used to compute the electrostatic interactions in the twodimensionally periodic geometry adopted here. Specifically, the dimension vertical to electrode of the simulation box was set to be 5 times the electrode separation,[43] which is sufficient to ensure that the accuracy of electrostatic force calculation is comparable to that of the twodimensional Ewald method. An FFT grid spacing of $0.1 \mathrm{~nm}$ and cubic interpolation for charge distribution were used to compute the electrostatic interactions in reciprocal space; a cutoff distance of $1.1 \mathrm{~nm}$ was used in the calculation of electrostatic interactions in the real space. The non-electrostatic interactions were computed by direct summation with a cutoff length of $1.1 \mathrm{~nm}$. 
The LINCS algorithm[44] was used to maintain bond lengths in the Emim ${ }^{+}$and $\mathrm{Tf}_{2} \mathrm{~N}^{-}$ions. Each simulation was started at $1000 \mathrm{~K}$ and subsequently annealed gradually to $298 \mathrm{~K}$ in $9 \mathrm{~ns}$. Following annealing, the system was simulated at that temperature for another 9 ns to reach equilibrium and then a 40-ns production run was performed for analysis.

\subsection{Results and Discussion}

The experiments were performed with room temperature ionic liquid (RTIL) 1-ethyl-3methyl-imidazolium bis(trifluoromethanesulfonyl)imide $\left(\mathrm{Emim}^{+} \mathrm{Tf}_{2} \mathrm{~N}^{-}\right)$on an atomically flat freshly-cleaved highly-oriented pyrolitic graphite (HOPG) surface. HOPG was chosen as a model system due to its direct relevance to carbon-based electrodes for electrochemical capacitors. As a first object, we explore the perturbation in the layered structure introduced by well-defined structural defects, namely graphite step-edges. After each cleavage, the features on the HOPG surface will vary, and the surface contains regions of atomically smooth plateaus combined with cleavage steps (single or multilayer), dislocations, grain boundaries, graphite strands, fibers, clusters and small particles.[34] As practical materials will inevitably contain such surface defects, it is important to understand their effect on the structure of the electrical double-layer of ionic liquids, as it will undoubtedly affect the electrochemical properties. Figure 1a shows a topography image wherein force-distance curves were collected in 20 different locations across the HOPG surface in $25 \mathrm{~nm}$ increments normal to the direction of the step-edges to examine the effect of graphite step-edges on the ion layering at these surface defects. The top panel of Fig. 1b shows a bivariate histogram of 50 consecutively measured force-distance curves at a single location on the HOPG basal plane, far from a step-edge (spot 1), indicated in the topography image in Fig. 1a. At this location the force-distance curves have distinct segments 
associated with interaction of the AFM tip with the ion layers at the interface as observed in previous work on HOPG. ${ }^{4}$ The bottom panel of Fig. $1 \mathrm{~b}$ shows a histogram of the separation values for the measured force-distance curves (Fig. 1b), with the peak at 0 separation being assigned to the surface. In order to interpret the force distance curves and conclude on measured ion composition and orientation, the experimental data needs to be compared with theory as reported in Ref.[4]. It was found that the ion positions measured with the AFM tip coincide with the predicted positions of the anion layers for this ionic liquid. Here, it is assumed that this selectivity is still valid and we interpret the measured steps as anion layer positions. As result, the distance between measured ion layers is determined by the size of the ion pair which was calculated to be around $0.7 \mathrm{~nm}$.[4] Figure 1c is a top-down view of the separation value histograms for each of the 20 locations across the surface as indicated in Fig. 1a. At locations on the surface at/near the step-edge the force-distance curves are markedly different (e.g., locations at spot 6,13 , and 18). Figure 1d shows the bivariate histogram of force-distance curves and the histogram of the separation values for force-distance curves collected at an HOPG step-edge (spot 13). The force-distance curves in this location look very different from what is observed on the basal plane (e.g., spot 1). On the basal plane, several ion layers are observed extending ca. 3 $\mathrm{nm}$ from the carbon surface (Fig. 1b). At the step-edge however, no features are present in the force profile at separation values over 1nm (Fig. 1d), and only one ion layer is observed at ca. $0.37 \mathrm{~nm}$. 

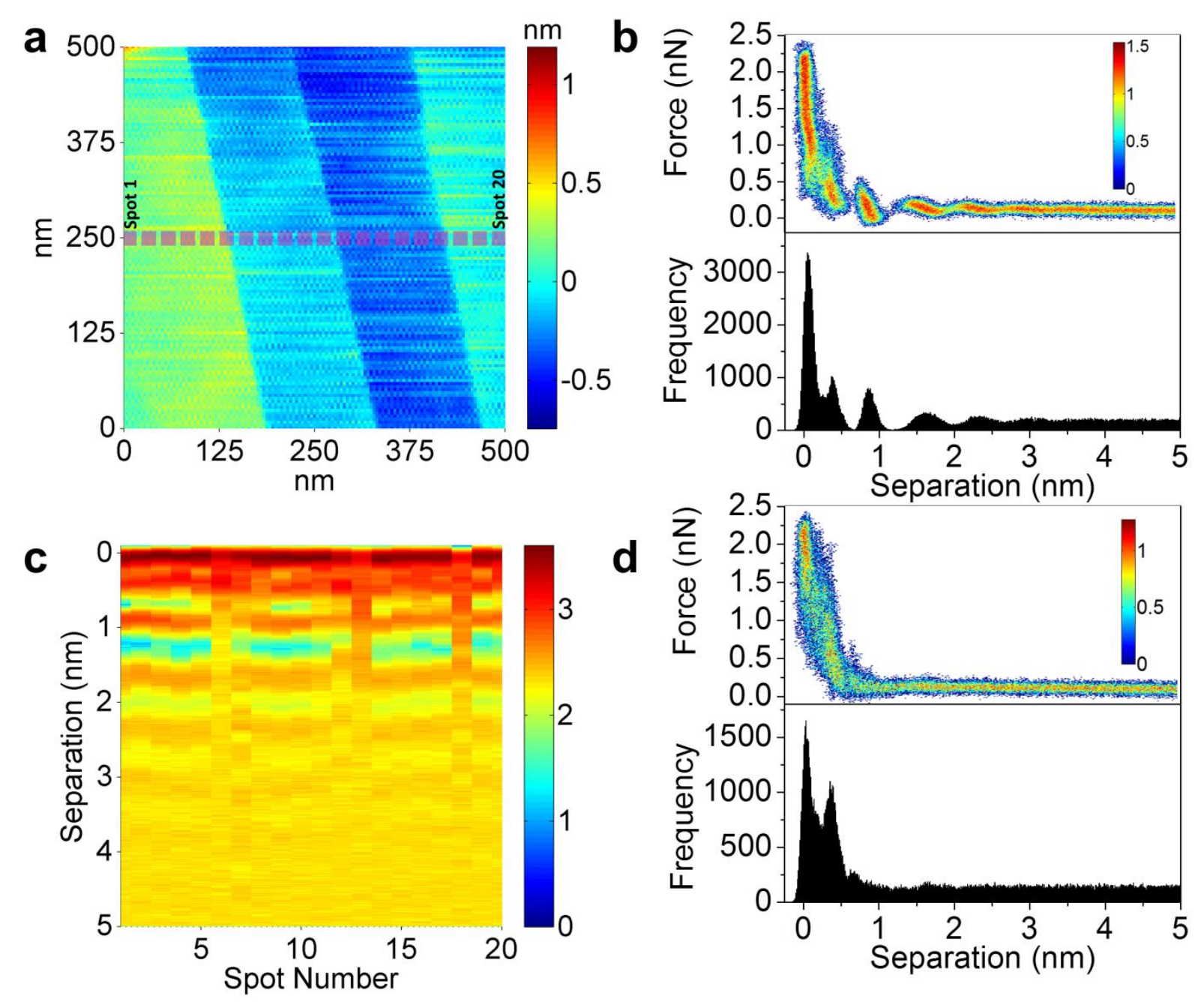

Figure 1: Force-distance curves at HOPG basal plane and step-edge. a) Topography of HOPG surface $(500 \times 500 \mathrm{~nm})$ showing location of 20 points where force-distance data were collected. b) Bivariate histogram of 50 measured force-distance curves and histogram of separation values collected on HOPG basal plane (spot 1 indicated in (a)). The color bar in the top panel represents the common logarithm of the frequency of data points in each bin. c) Top down view of separation histograms for all 20 locations, color bar represents the common logarithm of the frequency of data points at each separation value. d) Bivariate histogram of 50 measured force-distance curves and histogram of separation values collected at an HOPG step-edge (spot 13 indicated in (a)). Color bar is same as for (b).

These observations then raise the question of whether the presence of a step-edge on the graphite surface induces a disordering of the ionic liquid locally which is detected by the forcedistance measurement. To investigate the effect of the step-edge on the ionic liquid structure, molecular dynamics (MD) simulations were performed. Figure 2a shows a snapshot of the MD 
simulation in which a carbon step-edge is present. The cation and anion density profiles around the step-edge $(x=2.1 \mathrm{~nm})$ are shown in Fig. 2c. The near surface ion layer contains a mixture of cations and anions with alternating density of anions and cations extending further from the surface (e.g. the cation density profile at the lower carbon $\mathrm{x}<2.1 \mathrm{~nm}$ shows enhanced density at $\mathrm{z}$ values of ca. 0.55 and $1.25 \mathrm{~nm}$, while the anion shows enhanced density at ca. 0.8 and $1.5 \mathrm{~nm}$ ). At the step-edge there is a 'bending' of the ion layers to adjust to the new surface position, however the alternating arrangement of ions in the smectic-like structure is maintained. This 'bending' of the ion layers occurs over a small space of $<1 \mathrm{~nm}$. The MD results then suggest that there is no long-range disordering of the ion layers at the step-edge. 

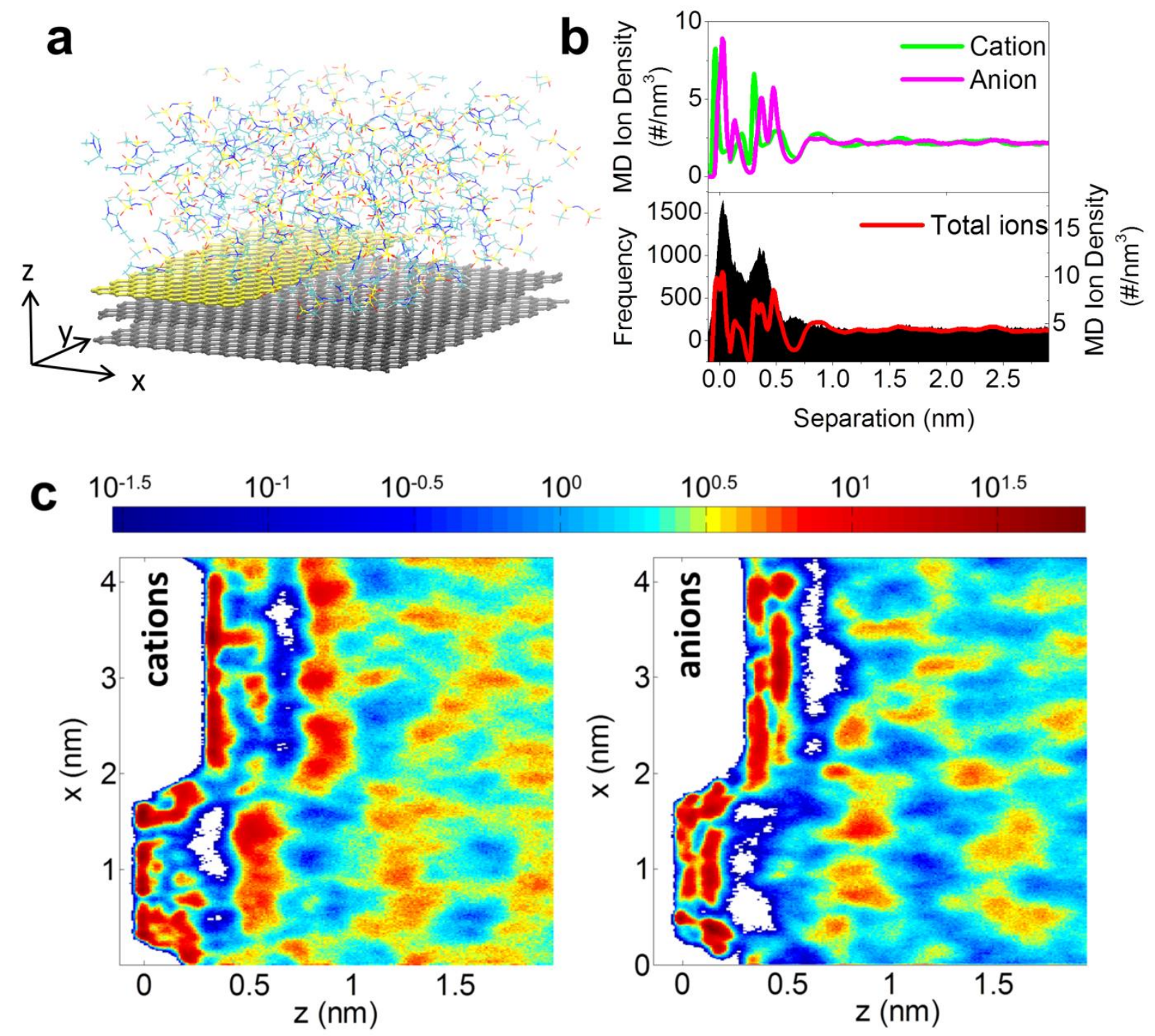

Figure 2: Molecular dynamics simulation of ionic liquid structure at carbon step-edge and comparison with experiment. a) Snapshot of molecular dynamics simulation containing carbon stepedge. b) Cation and anion density profile from MD averaged across the entire $\mathrm{x}$ range (top) and separation histogram from spot 13 together with the total number of ions from MD (bottom). c) Cation and anion number density (plotted on a log scale) as a function of distance from carbon surface (z) and position along carbon surface ( $\mathrm{x}$ - step-edge occurs at $2.1 \mathrm{~nm}$ ). In (b) and (d) the separation and $\mathrm{z}$ values are referenced to the carbon surface highlighted in yellow in part (a).

Since the AFM tip in the force-distance measurements is ca. $20 \mathrm{~nm}$ in radius the experimental force curves would be an averaged response of ion layers present on the first carbon surface and the second carbon surface which is shifted by the distance of the step height. Figure $2 \mathrm{~b}$ shows the cation and anion density profiles from MD averaged for all $\mathrm{x}$ values across 
the step-edge, as well as a separation histogram collected at one of the step-edges (spot 13) together with the number of total ions as a function of separation. The comparison of the separation histogram measured at the step-edge with the average number of total ions across the step show some similarity between them. The ion layer near 0 separation (adjacent to carbon surface at $x<2.1 \mathrm{~nm})$ coincides with the surface peak $(x>2.1 \mathrm{~nm})$, followed by a shoulder at ca. $0.13 \mathrm{~nm}$. The ion layer at ca. $0.4 \mathrm{~nm}$ (adjacent to carbon surface at $\mathrm{x}>2.1 \mathrm{~nm}$ ) is clearly observed in the ion density profile, however MD predicts that the variation of the density for the cation, anion, and the total ion density quickly drops off after this with no ion layering observed at larger separation values. This matches well with what is observed experimentally. Therefore, in conjunction with MD modeling, the experimental results can be interpreted as an averaged response of the ion layers from the two carbon surfaces. This demonstrates the lateral resolution of the technique. It is also important to note that the MD simulation used here neglects the possible presence of heteroatoms/functional groups which may be present on the carbon step edge after cleavage in air. It is known that different oxygen-containing functional groups can be present at edge-sites due to reaction with oxygen or water during cleavage,[35] and therefore it would be interesting to investigate the effect of functional groups on the local ion structure.

While the exploration of the RTIL ordering on the step-edges has revealed relatively minor changes in liquid structure, studies of the basal planes revealed serendipitous structures in the ordered layers. Figure 3a shows a line profile of the topography of the region on the HOPG surface that force-distance curves were measured at 100 different locations in $10 \mathrm{~nm}$ increments. Figure $3 \mathrm{~b}$ shows a top down view of the separation histograms collected at each location across the HOPG surface. In this location the HOPG surface does not contain single sharp step-edges as did the previous measurement, and rather contains a few large steps consisting of several carbon 
layers each (ca. 3-6 carbon layers per step). Here, the previously observed loss in segmentation of the force-distance profile at the step-edges is not observed. Across the observed region, several 'kinks' can be observed in the ordering of the ionic liquid. These are visible as gradually shifting lines highlighted in Fig. $3 \mathrm{~b}$ and happen over distances of $50 \mathrm{~nm}$ to $80 \mathrm{~nm}$ and can be described as an edge dislocation in the structure of the ionic liquid. This is analogous to typical defects observed in liquid crystals.[36,37] The dislocations are observed in regions with no stepedges (e.g. Region I and II) or can be associated with changes in the underlying topography (e.g. Region III). At the beginning and end of the dislocation region the histograms are very similar with sharp peaks and ion layers occurring at the same positions. In the center of this region the ion layers are shifted in position. At the start of Region I (spot 53) the peak for the first ion layer is located at $0.35 \mathrm{~nm}$ separation. Throughout Region I this peak begins shifting closer to the surface and combining with the surface peak at $0 \mathrm{~nm}$, while the peaks associated with the other ion layers shift closer to the surface. The first ion layer eventually disappears and the second ion layer now occupies the location that the first layer did previously.

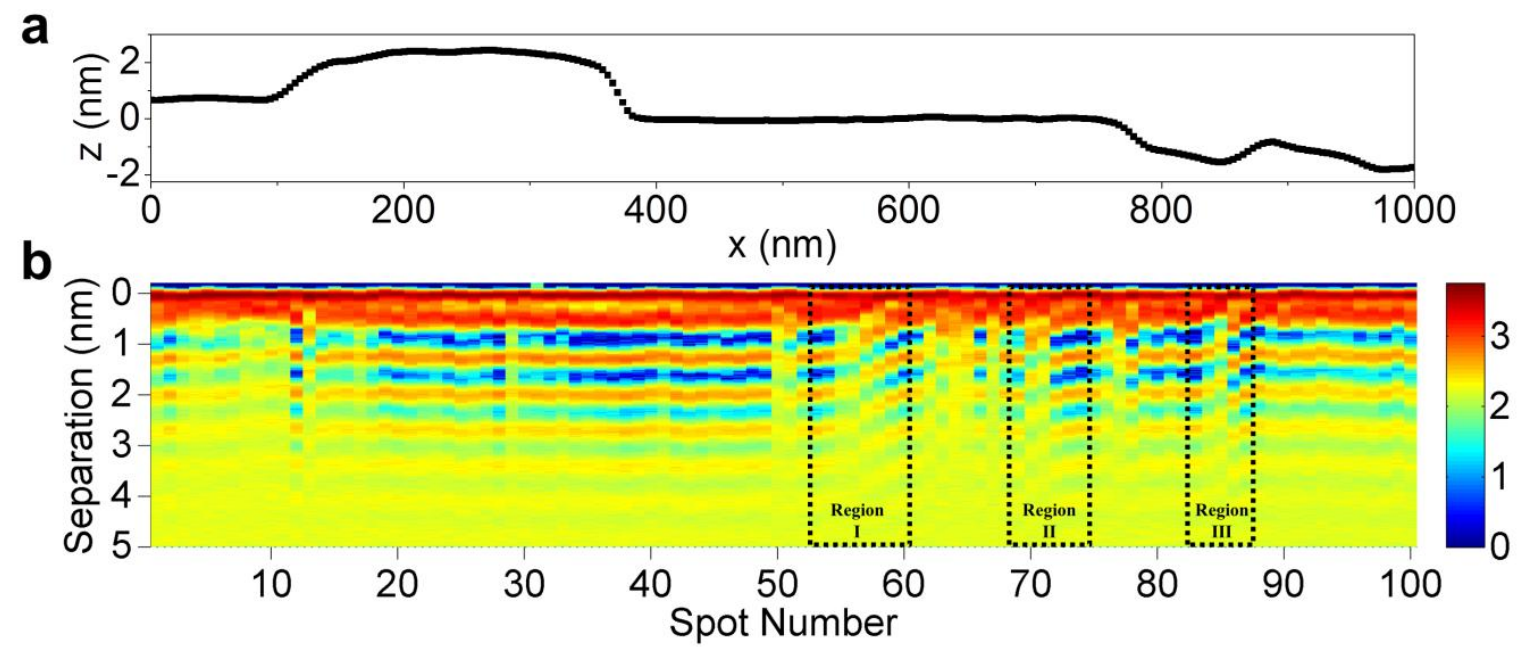

Figure 3: Force-distance curves across HOPG surface showing multiple defects in ion layer structure. a) Line profile of topography of HOPG surface where 100 sets of force-distance curves were measured in $10 \mathrm{~nm}$ increments. b) Top down view of separation histograms for all 100 locations, color 
bar represents the common logarithm of the frequency of data points at each separation value. Regions IIII mark the location of observed dislocation defects.

To quantify the structural changes in the ionic liquid along the observed edge dislocations, the separation histograms were fit using several Gaussian functions, where each function represents one ion layer. Figure 4a shows a detailed view of Region I. Figure 4b shows the separation histogram for the first location of Region I (spot 53) as well as the Gaussian functions used to fit the experimental data. The position and width of the fitted Gaussian peaks (i.e., the full width at half maximum, FWHM) for each location (spot 53-60) are shown in Fig. 4c and d, respectively. Across the dislocation the position of the ion layers gradually move closer toward the surface until the ion layer closest to the surface disappears (Fig. 4c). During this transition the width of the peaks increase, going through a maximum and ultimately ending close to the original value (Fig. 4d). The peak attributed to the surface layer has a nearly constant width across each of the defects consistent with this peak being from the interaction of the AFM tip with the HOPG surface as opposed to an ion layer. Here, the width of the peak can be interpreted as a variation in degree of ion ordering within the ion layer: A strong layering results in a very sharp well-defined ion layer position, whereas a high degree of disordering results in an ill-defined ion layer position and thus a broad peak in the force-distance curve histograms. This highlights the need to measure enough curves for statistical significance. This is the first time the degree of ion ordering can be directly visualized and analyzed through imaging techniques with a lateral resolution of around $20 \mathrm{~nm}$. This will allow for comparison of the AFM result with X-ray and neutron scattering techniques performed on similar systems in the future.[16, 38] All the dislocations marked in Region I-III show the same general behavior but it happens over different lengths scales between 50 and $80 \mathrm{~nm}$. Since these features are quite broad we are able to easily resolve them using the AFM measurements, whereas sharper features such as those associated 
with single step-edges on the carbon surface are at the lateral resolution limit of this technique. The different length scales over which the defects in the ion layering occur could be attributed with different formation energies. However, they all can be described using a universal curve when the data is plotted as function of distance between ion layer and surface instead of physical position as shown in Fig. 5.
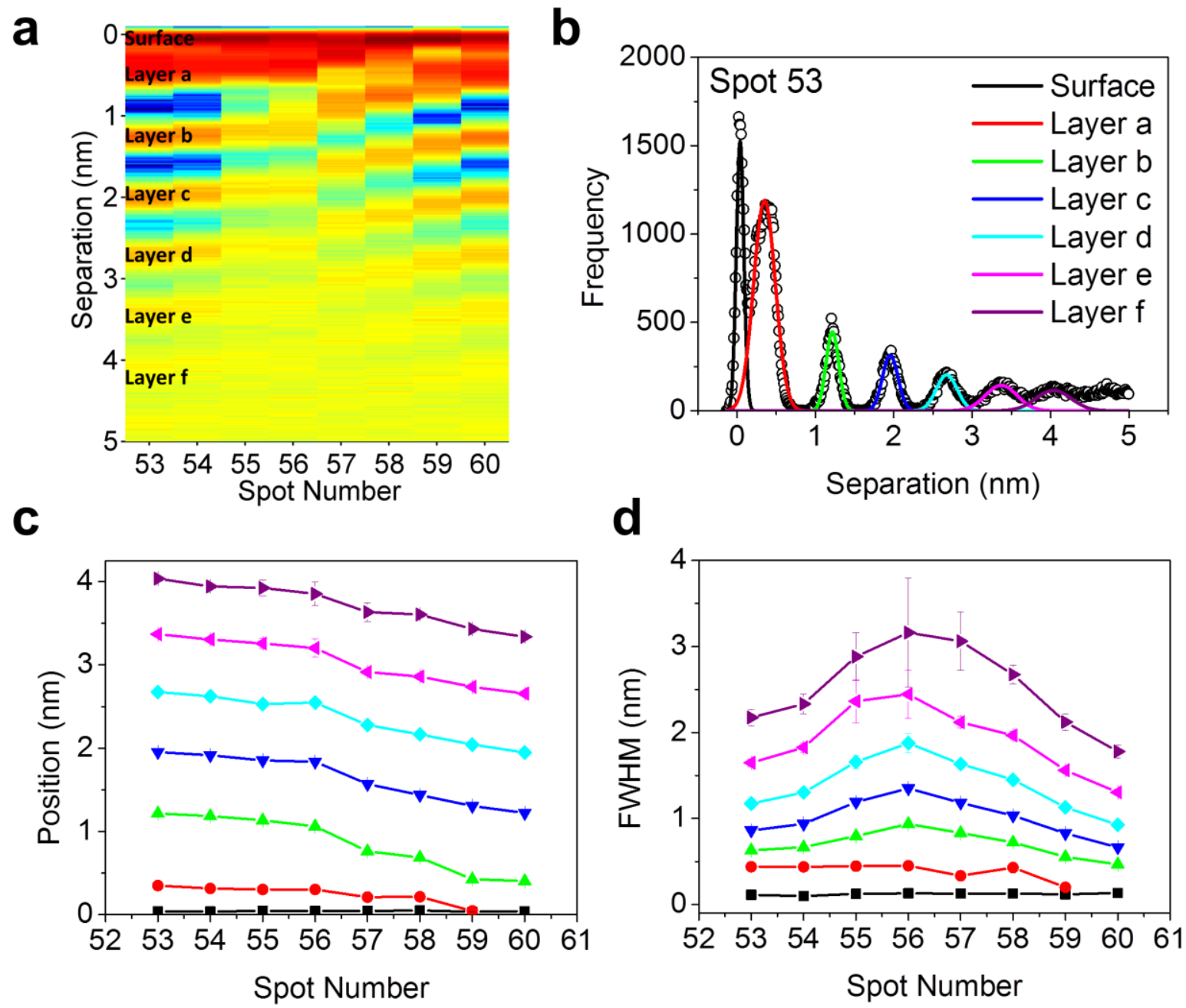

Figure 4: Analysis of single dislocation defect in ion layer structure. a) Top down view of separation histograms for locations 53-60, color intensity plotted on a log scale. b) Separation histogram for spot 53 with Gaussian functions used to fit data. Position (c) and full width at half maximum (offset for better visualization, same data without offset is shown in Fig. 5) (d) of Gaussian functions used to fit separation histograms shown in a. Legend for (c) and (d) same as (b). 


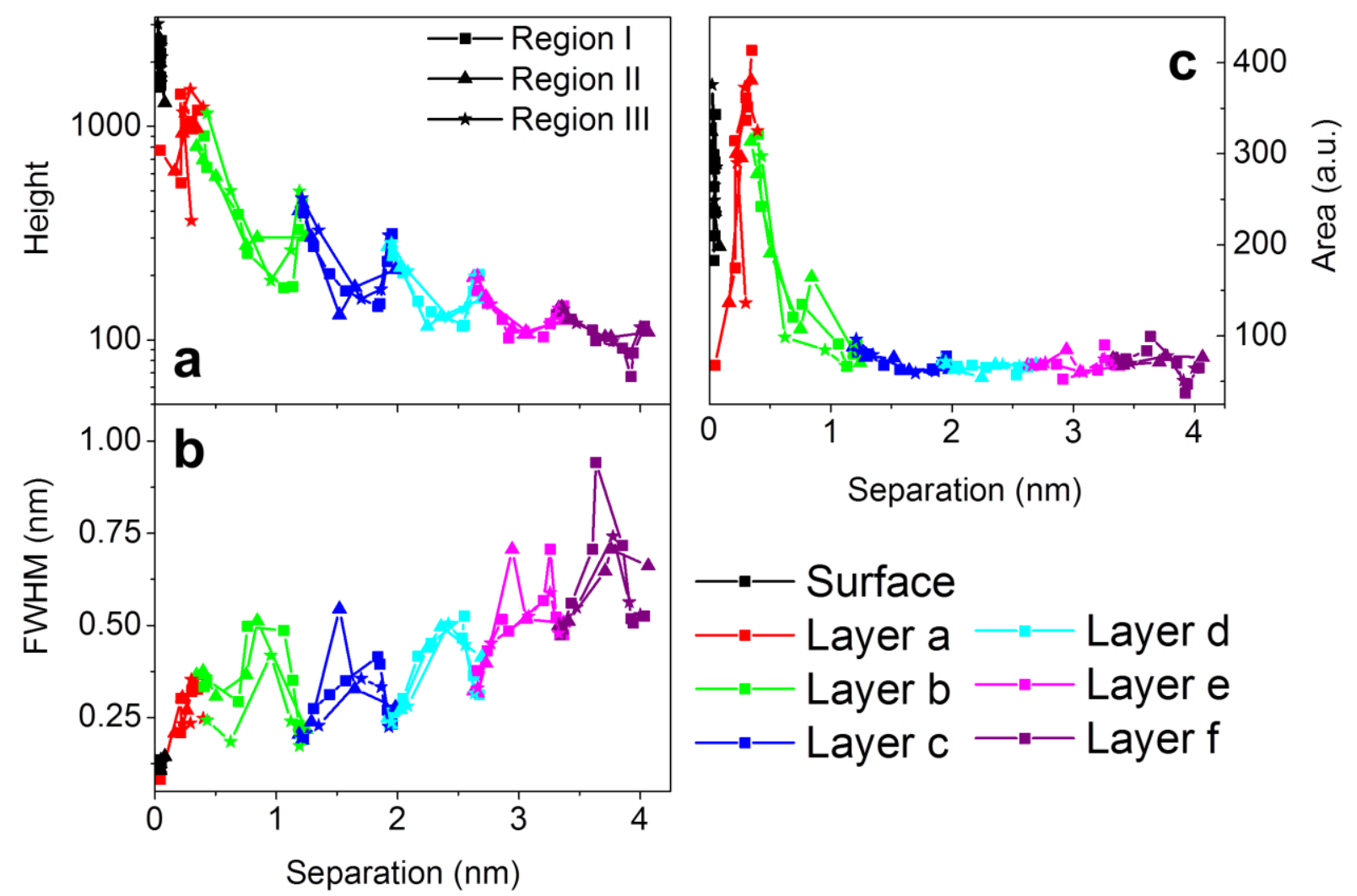

Figure 5: Universal behavior of force-distance curves within all defects. Height plotted on a log scale (a), full width at half maximum (b) and area (c) of Gaussian functions used to fit separation histograms as a function of distance to surface for Regions I-III.

The peak height, FWHM, and area of the fitted peaks for each ion layer as a function of peak position (separation) for all locations in Regions I-III fall upon universal curves. In the plot of the peak height vs. position (Fig. 5a) there is a general trend of increasing peak height as we move toward the surface with local maximum along the curve at ca. $0.25 \mathrm{~nm}, 1.22 \mathrm{~nm}, 1.94 \mathrm{~nm}$, $2.67 \mathrm{~nm}$, and $3.37 \mathrm{~nm}$. Note that these values of ion positions are slightly different than shown in Fig. 1. This is due to the fact that the data in Fig. 1 was measured using an uncoated $\mathrm{Si}_{3} \mathrm{~N}_{4}$ tip, whereas a gold coated tip was used for the data presented in Fig. 2-5. The role of tip chemistry in measured ion layering using AFM is an ongoing discussion and the subject of further research. However, it does not change the observed defects in ion layering and data interpretation. The 
trend of increasing peak height as we move toward the surface can be explained by an increase in ion density close to the surface (illustrated in Fig. 2c). A higher ion density will require a larger applied force on the AFM tip to break through the layer thereby causing an increase in the peak height at small separation values. Similarly, the peak width vs. separation curve has a generally increasing trend moving away from the surface with local minima at the locations of the local maxima in the height vs. separation curve. The increase in peak width moving away from the surface can be seen as a measure of the decreased ordering moving away from the surface, as the RTIL transitions from the ordered LC-like structure at the interface to the isotropic behavior of the bulk. The positions of the local minima in the width profile (or maxima in the height profile) are considered as the equilibrium position of the ion layers (i.e., the low energy positions). Within the edge dislocation of the ionic structure the ion layers shift from their equilibrium position to a higher energy position, in which the layered structure is less ordered, resulting in an increased peak width and decreased peak height. The peak area as a function of position increases rapidly as we move toward the surface until a separation of ca. $0.25 \mathrm{~nm}$ at which point it quickly drops off until the surface is reached. This dimension matches closely with the smallest dimension of the ions of the ionic liquid $\left(\mathrm{Emim}^{+}-0.85 \mathrm{~nm} \times 0.55 \mathrm{~nm} \times 0.28 \mathrm{~nm}, \mathrm{Tf}_{2} \mathrm{~N}^{-}\right.$$1.09 \mathrm{~nm} \times 0.51 \mathrm{~nm} \times 0.47 \mathrm{~nm}) .{ }^{4}$ The increase in area can be explained in the same way as the general increase in peak height since the ion layers become more ordered toward the surface and therefore require more force to puncture.[39]

\subsection{Conclusions}

To summarize, we have observed the internal structure of topological and structural defects on the ionic liquid-HOPG interface using 3D force mapping. Single step edges were 
found to result only in short range changes in ionic liquid ordering, with the changes in 3D force fields consistent with a rigid shift of the layers with narrow disordered region between the two surfaces, consistent with MD simulations. At the same time, formation of serendipitous dislocation-type topological defects were observed on planar surfaces. The decrease in the ordering was observed within the defect, consistent with behavior expected for classical liquid crystals. This is the first time, dislocations in an ordered liquid system could be directly visualized with a lateral resolution of down to $20 \mathrm{~nm}$. By performing statistics on force-distance curves we are able to measure the degree of disorder in each individual ion layer qualitatively which allows comparing AFM results with scattering techniques forming a cross-cut research opportunity. These studies offer a universal pathway for probing the internal structure of topological defects in soft condensed matter on length scales comparable (10-100 nm) but above those of MD. In the future, force-distance curve mapping will be used to investigate structure and dynamics of topological defects and their response to external fields during charging and discharging. Through this, we will gain a fundamental understanding of the influence of extended defects in the electric double layer on the charge storage performance.

\section{Acknowledgements}

The experimental and modeling efforts of JB, GF, and PTC were supported by the Fluid Interface Reactions, Structures and Transport (FIRST), an Energy Frontier Research Center funded by the U.S. Department of Energy, Office of Science, Office of Basic Energy Sciences. Additional personal support was provided by the U.S. Department of Energy, Basic Energy Sciences, Materials Sciences and Engineering Division through the Office of Science Early 
Career Research Program (NB) and the Center for Nanophase Materials Sciences, which is a DOE Office of Science User Facility. (MBO and SVK). We thank the computational resource from the National Energy Research Scientific Computing Center, which is supported by the Office of Science of the U.S. Department of Energy under Contract No. DE-AC02-05CH11231. G.F. also acknowledges the support from National Natural Science Foundation of China (51406060). 


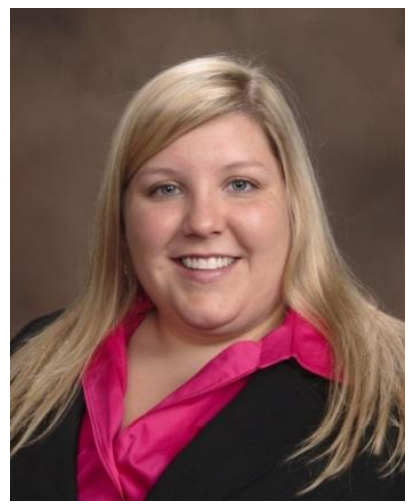

Jennifer Black is a postdoctoral research associate at the Center for Nanophase Materials Sciences (CNMS) at Oak Ridge National Laboratory. She did her Ph.D. at Dalhousie University focusing on the self-discharge mechanisms of electrochemical double-layer capacitors. She then worked as a postdoctoral researcher at the Ohio State University researching anode materials for lithium ion batteries and developing metallic foams for energy storage/conversion. She joined Oak Ridge National Laboratory in 2012 where her current research focuses on the nanoscale characterization of energy storage materials using scanning probe microscopy.

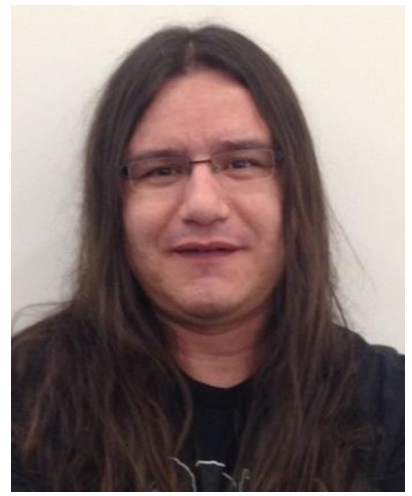

M. Baris Okatan received his B.S. and M.S. degrees in materials and metallurgical engineering from the Middle East Technical University, Ankara, Turkey, respectively in 2003 and 2006 and his Ph.D. degree in materials engineering from University of Connecticut, Storrs, CT in 2011. From 2011 to 2013 he worked in the University of New South Wales, Sydney, Australia as a postdoctoral research associate and then joined Oak Ridge National Laboratory as a postdoctoral research associate where he focuses on data visualization and analysis in materials science.

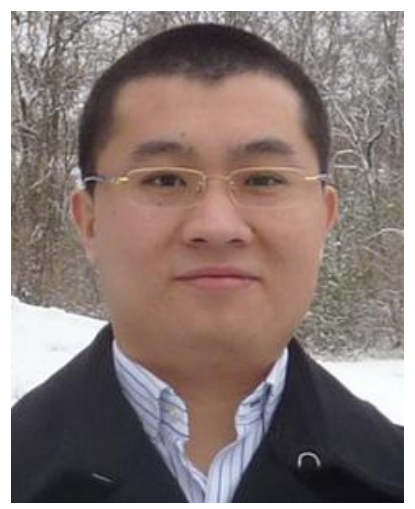

Guang Feng is a professor in Huazhong University of Science and Technology (HUST), awarded by the Hubei Provincial 100 Talents Program. He received his B.S. (2002) and M.S. (2005) from HUST and Ph.D. (2010) from Clemson University. From 2010 to 2013 he worked in Vanderbilt University and the Fluid Interface Reactions, Structures and Transport (FIRST) Energy Frontier Research Center as a postdoctoral research associate and then a research assistant professor. His research focuses on micro-/nano-scale interface and transport phenomena in capacitive energy storage, gas storage, and biological nanochannel. 

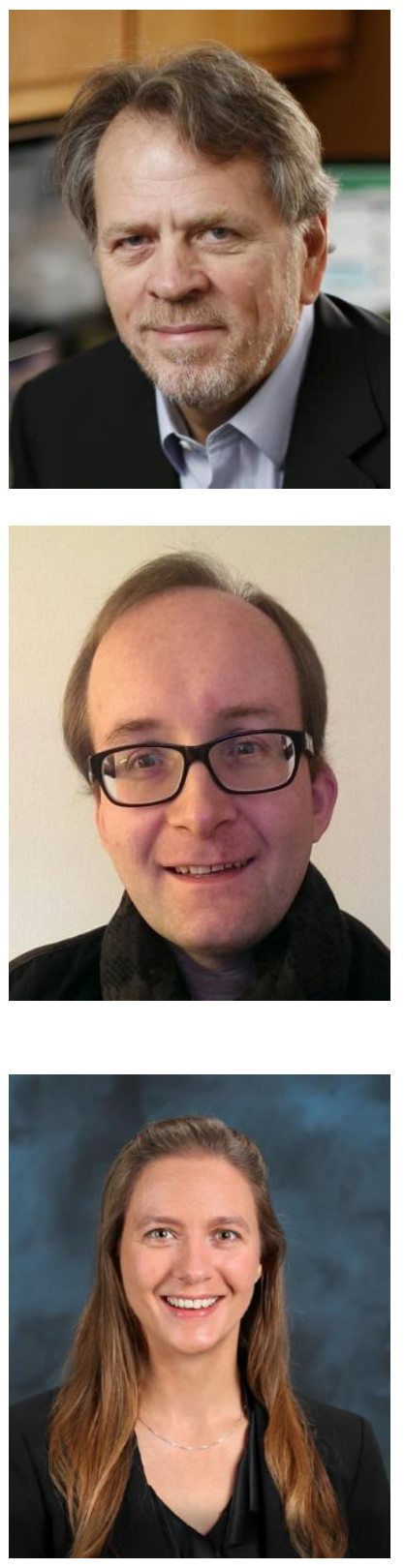

Peter T. Cummings, the John R. Hall professor of chemical engineering at Vanderbilt University (VU), also holds the position of Associate Dean for Research in the VU School of Engineering. For 20 years (1994-2013), he was associated with Oak Ridge National Laboratory (ORNL) at $40-50 \%$ effort. From 2007-13, he served as the chief scientist in ORNL's Center for Nanophase Materials Sciences (CNMS). His research interests include statistical mechanics, computational materials science, computational and theoretical nanoscience. He is the author of over 380 refereed journal publications and the recipient of many awards. He is fellow of APS, AAAS and AIChE.

Sergei V. Kalinin is the director of the Institute for Functional Imaging of Materials (IFIM) and a distinguished research staff member at the Center for Nanophase Materials Sciences (CNMS) at Oak Ridge National Laboratory (ORNL). He received his PhD from the University of Pennsylvania in 2002, followed by Wigner fellowship at ORNL. He is the recipient of many international awards including the Presidential Early Career Award for Scientists and Engineers and the Burton medal of Microscopy Society of America. He studies the coupling between electromechanical, electrical and transport phenomena on the nanoscale and applications of big, deep, and smart imaging data in materials sciences.

Nina Balke is a research staff member in the Center for Nanophase Materials Sciences (CNMS) at Oak Ridge National Laboratory. In 2006 she received her Ph.D in Materials Sciences from the Technical University of Darmstadt, Germany. After being a Feodor-Lynen fellow at the University of California in Berkeley she transitioned to CNMS in 2009 and is now working on nanoscale characterization of electromechanical effects in oxides focusing on energy storage materials. Her work was awarded the Department of Energy Early Career Research Award in 2011 and the American Ceramic Society's Robert L. Coble Award for Young Scholars in 2013.

\section{$\underline{\text { TOC Graphic }}$}
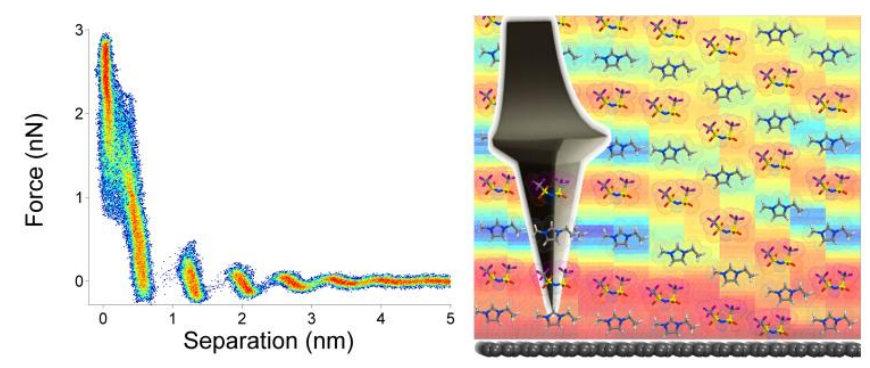


\section{References}

[1] M. Galiński, A. Lewandowski, I. Stępniak, Electrochim. Acta. 51 (2006) 5567-5580.

[2] A.A. Kornyshev, J. Phys. Chem. B. 111 ( 2007) 5545-5557.

[3] M.Z. Bazant, B.D. Storey, A.A. Kornyshev, Phys. Rev. Lett. 106 (2011) 046102.

[4] J.M. Black, D. Walters, A. Labuda, G. Feng, P.C. Hillesheim, S. Dai, P.T. Cummings, S.V. Kalinin, R. Proksch, N. Balke, Nano Lett. 13 (2013) 5954-5960.

[5] R. Hayes, S.Z. El Abedin, R. Atkin, J. Phys. Chem. B. 113 (2009) 7049-7052.

[6] R. Atkin, N. Borisenko, M Drueschler, F. Endres, R. Hayes, B. Huber, B. Roling, J. Mol. Liq. 192 (2014) 44-54.

[7] X. Zhang, Y.X. Zhong, J.W. Yan, Y.Z. Su, M. Zhang, B.W. Mao, Chem. Commun. 48 (2012) 582-584.

[8] R. Hayes, N. Borisenko, M.K. Tam, P.C. Howlett, F. Endres, R. Atkin, J. Phys. Chem. C. 115 (2011) 6855-6863.

[9] F. Endres, N. Borisenko, S.Z. El Abedin, R. Hayes, R. Atkin, Faraday Discuss. 154 (2012) 221-233.

[10] R. Atkin, G.G. Warr, J. Phys. Chem. C. 111 (2007) 5162-5168.

[11] R. Atkin, N. Borisenko, M. Drueschler, S.Z. El Abedin, F. Endres, R. Hayes, B. Huber, B. Roling, Phys. Chem. Chem. Phys. 13 (2011) 6849-6857.

[12] R. Atkin, S.Z. El Abedin, R. Hayes, L.H.S. Gasparotto, N. Borisenko, F. Endres, J. Phys. Chem. C. 113 (2009) 13266-13272.

[13] I. Bou-Malham, L. Bureau, Soft Matter. 6 (2010) 4062-4065.

[14] M. Mezger, H. Schröder, H. Reichert, S. Schramm, J.S. Okasinski, S. Schöder, V. Honkimäki, M. Deutsch, B.M. Ocko, J. Ralston, M. Rohwerder, M. Stratmann, H. Dosch, Science 322 (2008) 424428.

[15] E. Sloutskin, R. M. Lynden-Bell, S. Balasubramanian, M. Deutsch, J. Chem. Phys. 125 (2006) 174715.

[16] A. Uysal, H. Zhou, G. Feng, S.S. Lee, S. Li, P. Fenter, P.T. Cummings, P.F. Fulvio, S. Dai, J.K. McDonough, Y. Gogotsi, J. Phys. Chem. C. 118 (2014) 569-574.

[17] S. Perkin, Phys. Chem. Chem. Phys. 14 (2012) 5052-5062.

[18] S. Wang, S. Li, Z. Cao, T. Yan, J. Phys. Chem. C. 114 (2010) 990-995.

[19] R. M. Lynden-Bell, M. Del Pópolo, Phys. Chem. Chem. Phys. 8 (2006) 949-954.

[20] G. Feng, J.S. Zhang, R. Qiao, J. Phys. Chem. C. 113 (2009) 4549-4559.

[21] G. Feng, R. Qiao, J. Huang, S. Dai, B.G. Sumpter, B. G. V. Meunier, Phys. Chem. Chem. Phys. 13 (2011) 1152-1161.

[22] M. V. Fedorov, A.A. Kornyshev, J. Phys. Chem. B. 112 (2008) 11868-11872.

[23] M. V. Fedorov, A.A. Kornyshev, Chem. Rev. 114 (2014) 2978-3036.

[24] C. Merlet, D.T. Limmer, M. Salanne, R. van Roij, P.A. Madden, D. Chandler, B. Rotenberg, J. Phys. Chem. C. 118 (2014) 18291-18298.

[25] A.A. Kornyshev, R. Qiao, J. Phys. Chem. C. 118 (2014) 18285-18290.

[26] G.B. Pan, W. Freyland, Chem. Phys. Lett. 427 (2006) 96-100.

[27] Y. Jeon, D. Vaknin, W. Bu, J. Sung, Y. Ouchi, W. Sung, D. Kim, Phys. Rev. Lett. 108 (2012) 055502.

[28] T. Carstens, R. Gustus, O. Hofft, N. Borisenko, F. Endres, H. Li, R.J. Wood, A.J. Page, R. Atkin, J. Phys. Chem. C. 118 (2014) 10833-10843.

[29] A.J. Page, A. Elbourne, R. Stefanovic, M.A. Addicoat, G.G. Warr, K. Voitchovsky, R. Atkin, Nanoscale 6 (2014) 8100-8106.

[30] J.J. Segura, A. Elbourne, E.J. Wanless, G.G. Warr, K. Voitchovsky, R. Atkin, Phys. Chem. Chem. Phys. 15 (2013) 3320-3328.

[31] K. Suzuki, N. Oyabu, K. Kobayashi, K. Matsushige, H. Yamada, Appl. Phys. Express 4 (2011) 125102. 
[32] K. Kimura, S. Ido, N. Oyabu, K. Kobayashi, Y. Hirata, T. Imai, H. Yamada, J. Phys. Chem. 132 (2010) 194705.

[33] T. Hiasa, K. Kimura, H. Onishi, M. Ohta, K. Watanabe, R. Kokawa, N. Oyabu, K. Kobayashi, H. Yamada, J. Phys. Chem. C. 114 (2010) 21423-21426.

[34] H. Chang, A. Bard, Langmuir 7 (1991) 1143-1153.

[35] R. McCreery, Chem. Rev. 108 (2008) 2646-2687.

[36] S. Jain, S. Defects and Order in Liquid Crystal Phases. Harvard University, 1999.

[37] P. Oswald, P. Pieranski, Smectic and Columnar Liquid Crystals: Concepts and Physical Properties Illustrated by Experiments. Taylor \& Francis, 2005.

[38] A. Uysal, H. Zhou, G. Feng, S.S. Lee, S. Li, P.T. Cummings, P.F. Fulvio, S. Dai, J. k. McDonough, Y. Gogotsi, P. Fenter J. Phys. Condes. Matter. 27 (2015) 032101-032109.

[39] M. Nobili, G. Durand, Phys. Rev. A. 46 (1992) R6174-R6177.

[40] O. Borodin, J. Phys. Chem. B. 113 (2009) 11463-11478.

[41] E. Lindahl, B. Hess, D. van der Spoel, J. Molec. Model. 7 (2001) 306-317.

[42] I.C. Yeh, M.L. Berkowitz, J. Chem. Phys. 111 (1999) 3155-3162.

[43] T. Darden, D. York, L. Pedersen, J. Chem. Phys. 98 (1993) 10089-10092.

[44] B. Hess, H. Bekker, H.J.C. Berendsen, J.G.E.M. Fraaije, J. Comput. Chem. 18 (1997) 1463-1472. 

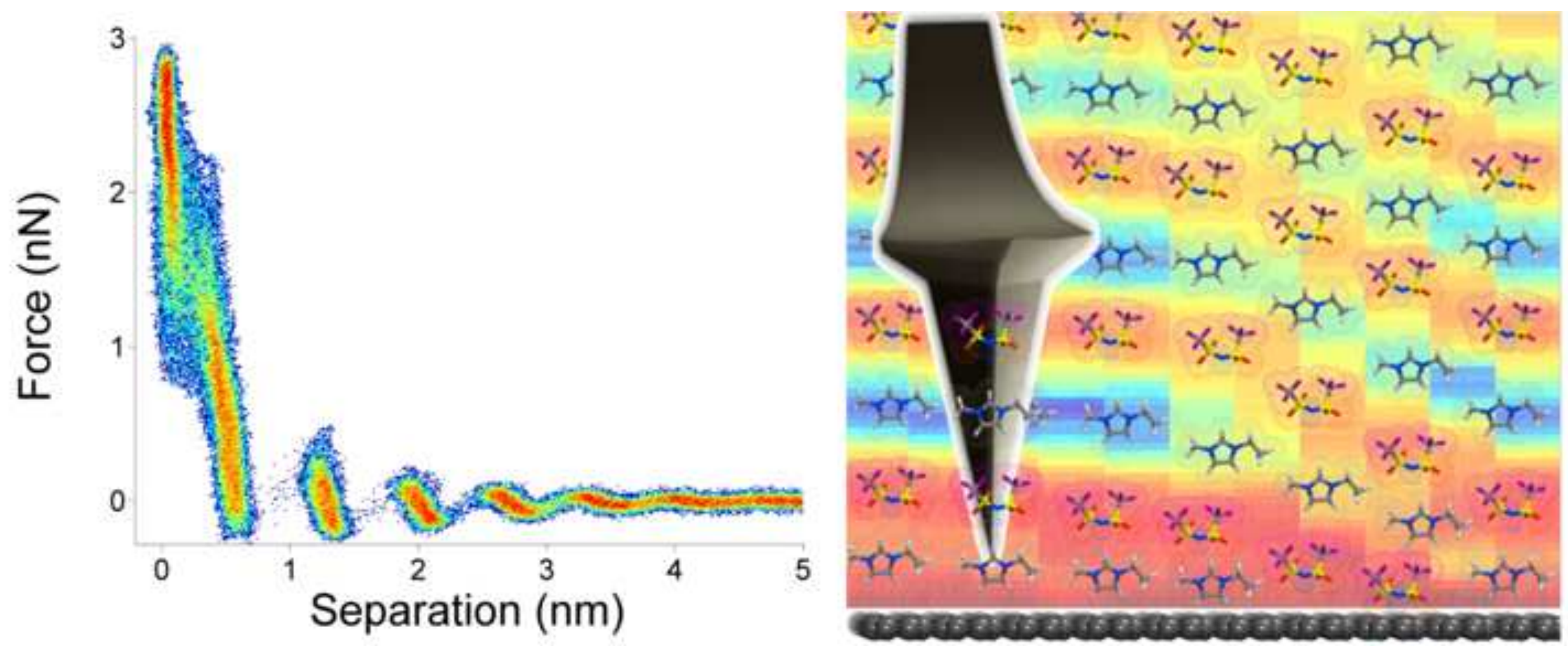